\title{
Peiminine ameliorates bleomycin-induced acute lung injury in rats
}

\author{
HAI GUO ${ }^{1}$, FUZHI JI ${ }^{1}$, BAORUI LIU ${ }^{2}$, XIAOFEI CHEN ${ }^{1}$, JINGDONG HE ${ }^{1}$, \\ XIAOFENG ZHAO ${ }^{1}$ and JIENING GONG ${ }^{3}$ \\ ${ }^{1}$ Cancer Center, Huai'an First People's Hospital, Huai'an 223300; ${ }^{2}$ Nanjing Medical University, Nanjing 210029; \\ ${ }^{3}$ Basic Medical College of Nanjing University of Traditional Chinese Medicine, Nanjing 210046, P.R. China
}

Received September 17, 2012; Accepted November 21, 2012

DOI: $10.3892 / \mathrm{mmr} .2013 .1312$

\begin{abstract}
The aim of this study was to investigate whether or not peiminine inhibits lung inflammation and pulmonary fibrosis in a rat model of bleomycin-induced lung injury. Rats were randomly divided into 4 groups. In 3 groups, intratracheal bleomycin $(5 \mathrm{mg} / \mathrm{kg})$ was used to induce acute lung injury, followed by administration of either carboxymethyl cellulose (control group, $n=14$ ), dexamethasone (DXS group, $\mathrm{n}=14$ ) or peiminine (peiminine group, $n=10$ ). In the fourth group (sham-operated, $n=12$ ), normal saline was instilled instead of bleomycin, followed by administration of carboxymethyl cellulose. Drugs were administered intragastrically for 28 days. Lung sections were stained with hematoxylin and eosin $(\mathrm{H} \& \mathrm{E})$ and Masson's trichrome, to grade the degree of alveolitis and pulmonary fibrosis. The lung index was calculated as the ratio of lung to body weight. Serum levels of interleukin-4 (IL-4), tumor necrosis factor- $\alpha$ (TNF- $\alpha$ ) and interferon- $\gamma(\mathrm{IFN}-\gamma)$ were obtained using a radioimmunoassay. Immunocytochemical methods were employed to assess the expression of transforming growth factor- $\beta$ (TGF- $\beta$ ), connective tissue growth factor (CTGF), NF- $\kappa$ B, extracellular signal-related kinase (ERK1/2), Fas and FasL in lung tissue. Peiminine and DXS significantly reduced alveolar inflammation and pulmonary interstitial inflammation in rats with bleomycin-induced lung injury. These protective effects were associated with significant $(\mathrm{P}<0.05)$ decreases in the levels of IFN- $\gamma$ in serum and of TGF- $\beta$, CTGF, ERK $1 / 2, N F-\kappa B$ and FasL in lung tissue. No effects were observed on serum TNF- $\alpha$ or IL-4. In conclusion, peiminine inhibits lung inflammation and pulmonary fibrosis in a rat model of bleomycin-induced lung injury, by reducing circulating IFN- $\gamma$ levels and inhibiting signal transduction pathways involving TGF- $\beta$, CTGF, ERK1/2, NF- $\kappa$ B and FasL.
\end{abstract}

Correspondence to: Dr Baorui Liu, Cancer Center, Huai'an First People's Hospital, Huai'an 223300, P.R. China

E-mail: baoruiliu@hotmail.com

Key words: pulmonary fibrosis, peiminine, inflammatory cytokines, cellular signal transduction pathways

\section{Introduction}

Esophageal, gastric and lung cancer are common diseases that pose a serious global threat to health. Chemotherapy agents such as bleomycin, mitomycin and methotrexate may cause pulmonary toxicity, while radiotherapy may lead to radiation pneumonitis $(1,2)$. Lung injury seriously hampers full implementation of treatment, limiting the potential benefits of therapy. The early phase of lung injury is characterized by inflammation (alveolitis), while the late phase is characterized by the organization and deposition of collagen with remodeling (pulmonary fibrosis) $(1,2)$.

The characteristic clinical and histological manifestations of acute lung injury (ALI) are initiated by a well-described network of cytokines $(3,4)$. The acute phase of ALI, characterized by alveolar inflammation, is mediated by tumor necrosis factor- $\alpha$ (TNF- $\alpha$ ), interleukin-1 (IL-1) and transforming growth factor- $\beta$ (TGF- $\beta$ ) $(3,4)$. In addition, interferon- $\gamma$ $(\mathrm{IFN}-\gamma)$ is able to maintain the inflammatory response in the lung by inducing macrophages to produce mediators, such as TNF- $\alpha$, IL-1, IL-6 and IL-8. TNF- $\alpha$ is an important signaling protein that is able to initiate and continually amplify local or systemic inflammatory responses (5).

TGF- $\beta$ is a key cytokine that induces lung injury and contributes to pulmonary fibrosis, through its actions to induce collagen gene expression or synthesis by stimulation of fibroblast proliferation $(3,4,6,7)$. Expression of the connective tissue growth factor (CTGF) gene acts as a downstream effector of TGF- $\beta 1$, and is thought to play an important role in pulmonary fibrosis through the promotion of extracellular matrix synthesis (8).

Numerous other signal transduction pathways have been implicated in the pathogenesis of pulmonary fibrosis. For example, the mitogen-activated protein kinase/extracellular signal-related kinase (MAPK/ERK) pathway is essential for the formation of pulmonary fibrosis $(9-11)$. NF- $\kappa \mathrm{B}$ is a significant transcription factor that is a key mediator of signal transduction during the acute inflammatory response and pulmonary fibrosis $(12,13)$.

Despite recent advances in our understanding of the epidemiology, pathogenesis and treatment of ALI, this condition remains a significant cause of morbidity and mortality in the critically ill patient population (14). At present, glucocorticoids are the most frequently used anti-inflammatory drugs for the 
clinical management of ALI $(9,15)$. However, currently there are no approved medical anti-fibrotic therapies (16), and hence the development of effective agents to ameliorate pulmonary fibrosis is urgently needed.

Peiminine is the main component of Fritillaria, and has been used for several years as a traditional Chinese medicine for a variety of conditions including pulmonary fibrosis. Peiminine has been reported to have effects as a relaxant of bronchial smooth muscle and as an antitussive $(17,18)$. In addition, there is evidence that alkaloids isolated from the Fritillaria bulb have anti-inflammatory, as well as antitussive actions $(17,18)$. However, to date there have been no studies exploring whether or not peiminine is able to inhibit pulmonary inflammation and fibrosis.

The aims of this study was to determine whether or not peiminine inhibits lung inflammation and pulmonary fibrosis in rats. Furthermore, the effects of peiminine on lung injury were correlated with changes in the levels of mediators implicated in the pathogenesis of ALI.

\section{Materials and methods}

Animals. Age- and gender-matched Sprague-Dawley (SD) rats (weight, 180-220 g) were purchased from the Experimental Animal Center of the Nanjing Medical University, kept in a 12-h dark/light cycle in a temperature- and humiditycontrolled room, and fed a standard laboratory diet and water. The experimental procedures were approved by the Animal Care and Use Committee of the Nanjing Medical University, China. Adequate measures were taken to minimize the pain experienced by the experimental animals.

Drugs and reagents. Peiminine (purity $>98 \%$ ) was obtained from Gamma Technology Development Co., Ltd. (Shenzhen China). Dexamethasone (DXS; $0.75 \mathrm{mg}$ ) was purchased from Tianjin Tianyao Pharmaceutical Co., Ltd. (Tianjin, China). Bleomycin ( $8 \mathrm{mg}$ ) was obtained from Tianjin Taihe Pharmaceutical Co., Ltd. (Tianjin, China).

Rabbit anti-rat polyclonal antibodies against TGF- $\beta$, CTGF, $\mathrm{NF}-\kappa \mathrm{B}, \mathrm{ERK} 1 / 2$, Fas and FasL were purchased from Beijing Zhongshan Golden Bridge Biotechnology Co., Ltd. (Beijing, China). IL-4 and TNF- $\alpha$ kits were provided by the Beijing Huaying Biotechnology Institute (Beijing, China). The IFN- $\gamma$ kit was obtained from the Beijing Huaying Biotechnology Institute, sourced from Adlitteram Diagnostic Laboratories, Inc. (West Palm Beach, FL, USA).

Instruments. The microtome was purchased from Leica (Mannheim, Germany). The optical microscope, Olympus DP71 microscope digital camera and fully automated image acquisition system were obtained from the Olympus Corporation (Tokyo, Japan).

The $\gamma$-911 automatic radioimmunoassay (RIA) counter was purchased from the Science and Technology Industrial Company of the China University, and the Stat Fax 2100 automatic microplate reader was purchased from Awareness Technology, Inc. (Palm City, FL, USA).

Experimental groups. Rats were randomly divided into 4 groups: the sham-operated $(n=12)$, the control $(n=14)$, the
DXS $(n=14)$ and the peiminine groups $(n=10)$. For the latter 3 groups, intratracheal administration of bleomycin was used to induce lung injury, to allow comparison of the effects of peiminine and DXS. For the sham-operated group, normal saline was applied instead of bleomycin, as the negative control for ALI.

Development of the ALI model in rats. After allowing adjustment to the environment, the rat was anesthetized with chloral hydrate $(10 \%)$ and fixed on a board in the supine position. For the control, DXS and peiminine groups, bleomycin $(5 \mathrm{mg} / \mathrm{kg})$ was instilled into the trachea of the rat using a microliter injector, based on methods described previously in the literature (19). For the sham-operated group, normal saline was administered instead of bleomycin. After intratracheal instillation of bleomycin or saline, the rat was placed in a vertical position and spun for $0.5 \mathrm{~min}$ to ensure that the solution was distributed evenly within the lungs.

Administration of drugs. Rats in the sham-operated and control groups were given 5\% carboxymethyl cellulose sodium (CMC) solution at a dosage of $1 \mathrm{ml} / 100 \mathrm{~g}$ weight; CMC was chosen as its viscosity was similar to that of the drugs used in the other 2 groups. Rats in the DXS group were given an equal volume of DXS solution at a dosage of $0.000405 \mathrm{~g} / \mathrm{kg}$ weight. Rats in the peiminine group were administered an equal volume of peiminine at a dosage of $0.005 \mathrm{~g} / \mathrm{kg}$ weight. Drugs were administered daily for 28 consecutive days, using gastric gavage; it has been reported previously that 28 days are required for the formation of lung fibrosis after administration of bleomycin (20).

Alveolitis and pulmonary fibrosis assay. Rats were anesthetized and sacrificed by carotid exsanguination. The left lung was fixed with $4 \%$ paraformaldehyde in phosphate-buffered saline (PBS) under 15-20- $\mathrm{cm} \mathrm{H}_{2} \mathrm{O}$ pressure. Lungs were embedded in paraffin, and $4-\mu \mathrm{m}$ sections were prepared. For histology, the sections were stained with hematoxylin and eosin (H\&E) and Masson's trichrome. To assay the severity of alveolitis and pulmonary fibrosis, the scoring method described by Szapiel et al (21) was used.

The grading criteria used for alveolitis were: 1 point, no alveolitis; 2 points, mild alveolitis, affecting $<20 \%$ of the total lung, showing infiltration of mononuclear cells into the widened alveolar septa, and limited to localized regions with involvement of nearby pleural areas; 3 points, moderate alveolitis, affecting an area of 20-50\%, with greater pleural involvement; 4 points, severe alveolitis, involving an area $>50 \%$, with occasional monocytes in the alveolar space and bleeding caused by consolidation.

The scoring criteria used for fibrosis were: 1 point, no fibrosis; 2 points, mild fibrosis, affecting an area $<20 \%$ of the whole lung, with fibrosis involving the pleura and subpleural interstitium, and disorders of alveolar structure; 3 points, moderate fibrosis, involving an area of $20-50 \%$, with localized areas of fibrosis extending from the pleura; 4 points, severe fibrosis, involving an area $>50 \%$, with fusion of alveolar spaces.

Lung index assay. Rats were anesthetized and sacrificed by carotid exsanguination, and their chest was opened to obtain the lungs. The trachea was removed and discarded, 


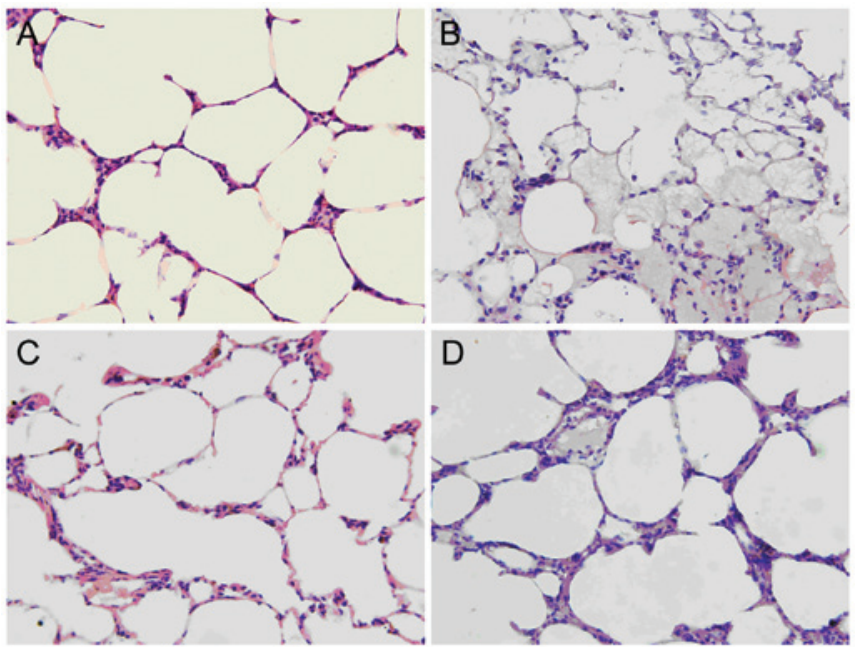

Figure 1. Section of lung stained with hematoxylin and eosin (H\&E) and observed under the light microscope. (A) Sham-operated group: the morphological structure was consistent with that of normal lung. (B) Control group: substantial pathological changes are evident, including widened alveolar septa, interstitial edema and inflammatory cell infiltration. (C) Dexamethasone group: although some pathological changes are evident, these are much less severe compared to those of the control group. (D) Peiminine group: the pathological changes are of a substantially lower degree to those observed in the control group.

and after drying the surface with filter paper, the lungs were weighed. The lung index was then calculated, based on lung weight and body weight: Lung index = lung weight $(\mathrm{g}) /$ body weight (g) x100\%

Assay for inflammatory cytokines. Rats were anesthetized and sacrificed by carotid exsanguination. The blood was collected, and centrifuged at 3,000 rpm for $10 \mathrm{~min}$ to obtain serum. The serum was subjected to RIA to determine the levels of IL-4, TNF- $\alpha$ and IFN- $\gamma$.

Assay of cell signal transduction pathways. The left lung was fixed with $4 \%$ paraformaldehyde in PBS under $15-20-\mathrm{cm}$ $\mathrm{H}_{2} \mathrm{O}$ pressure. The lung was embedded in paraffin and $4-\mu \mathrm{m}$ sections were prepared. Immunohistochemistry, using the streptavidin-biotin complex (SABC) method, with calculation of average optical density (IOD), was used to determine the levels of TGF- $\beta$, CTGF, NF- $\kappa$ B, ERK1/2, Fas and FasL.

Statistical analysis. Data are expressed as the means \pm standard deviation (SD). Statistical analyses were carried out using the SPSS 16.0 software. One-way analysis of variance (ANOVA) followed by the Student-Newman-Keuls test were used to compare the results in the various treatment groups. $\mathrm{P}<0.05$ was considered to indicate a statistically significant difference.

\section{Results}

General observations. In the initial period after surgical operation, rats in the control group (i.e., with bleomycin-induced ALI) had cold tails and limbs, dark purple tail veins and loss of hair luster. These symptoms gradually receded, showing improvement at 3 days, and had almost disappeared at 7 days. Rats in the control and DXS groups showed a reduced activity,

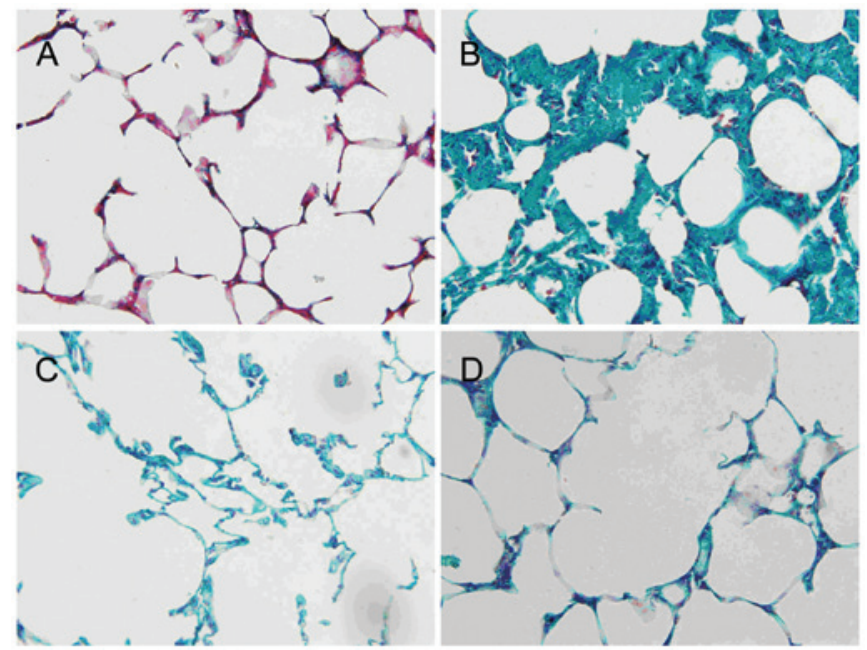

Figure 2. Section of lung stained with Masson's trichrome and observed under the light microscope. (A) Sham-operated group: only a small amount of collagen fibers are present. (B) Control group: there is a substantial increase in the number of collagen fibers, with a histological picture typical of pulmonary fibrosis. (C) Dexamethasone group: although fibrotic changes are evident, they are much less severe than those observed in the control group. (D) Peiminine group: the observed fibrotic changes are far less severe compared to those of the control group.

decreased appetite and weight loss. The weight loss was more evident in the DXS group; however, in these two groups, body weight gradually recovered over 18-21 days. In the shamoperated and peiminine groups, no noticeable reduction was observed in activity, appetite or weight. The mortality rates (during the 28-day period) in the control and DXS groups were $1 / 14$ rats. No rats died in the other two groups during this period.

Macroscopic observations of lung tissue. The lung tissue of the sham-operated group was pink, smooth and soft, with good elasticity. However, in the control group, a significant reduction was observed in the amount of normal lung tissue, with increasing occurrence of uneven pale foci, black lesions and reduced elasticity. In the DXS group, the extent of the lesions was smaller compared to the control group, although there was still a clear difference from the sham-operated group. The peiminine group showed no obvious changes, with lung tissue structure resembling that of the sham-operated group.

$H \& E$ staining observed under the light microscope. Representative examples of sections from the 4 groups are shown in Fig. 1. The morphological characteristics of the shamoperated group were consistent with those expected of normal lung structure. By contrast, the control group showed widening of the alveolar septa, interstitial edema and inflammatory cell infiltration into the pulmonary interstitium and the alveolar spaces. Although pathological changes were also evident in the DXS and peiminine groups, these lesions were less severe or extensive compared to those observed in the control group.

Masson's trichrome staining observed under the light microscope. Representative examples of sections from the 4 groups are shown in Fig. 2. In the lung tissue of sham-operated rats, a 

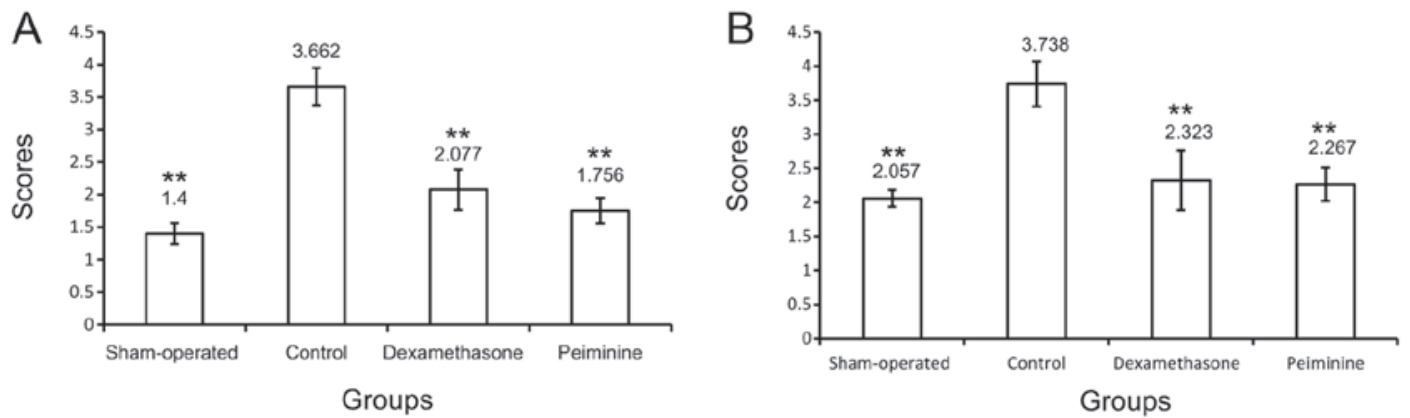

Figure 3. Alveolitis and pulmonary fibrosis scores. (A) Comparison of the alveolar inflammation scores for the 4 groups. The alveolitis score of the control group was significantly higher than that of the sham-operated group $(\mathrm{P}<0.01)$. The alveolitis scores of the dexamethasone and peiminine groups were significantly lower compared to that of the control group $(\mathrm{P}<0.01)$. (B) Comparison of the pulmonary interstitial inflammation scores for the 4 groups. The pulmonary fibrosis score of the control group was significantly higher compared to that of the sham-operated group $(\mathrm{P}<0.01)$. The pulmonary fibrosis score of the dexamethasone and peiminine groups were significantly lower compared to that of the control group $(\mathrm{P}<0.01) .{ }^{* *} \mathrm{P}<0.01$.

A

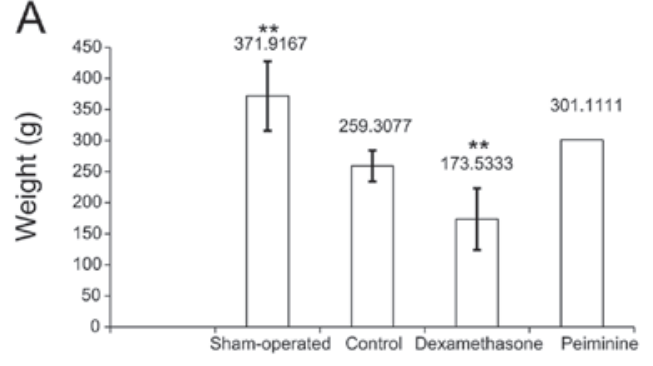

Groups

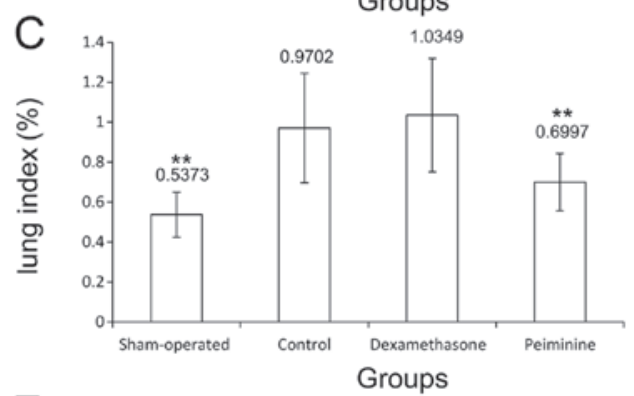

$\mathrm{E}$

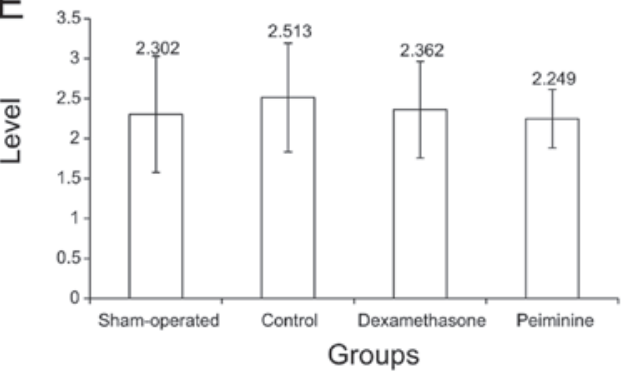

B

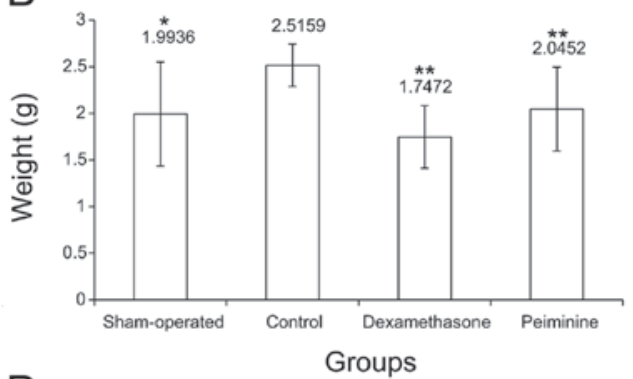

D
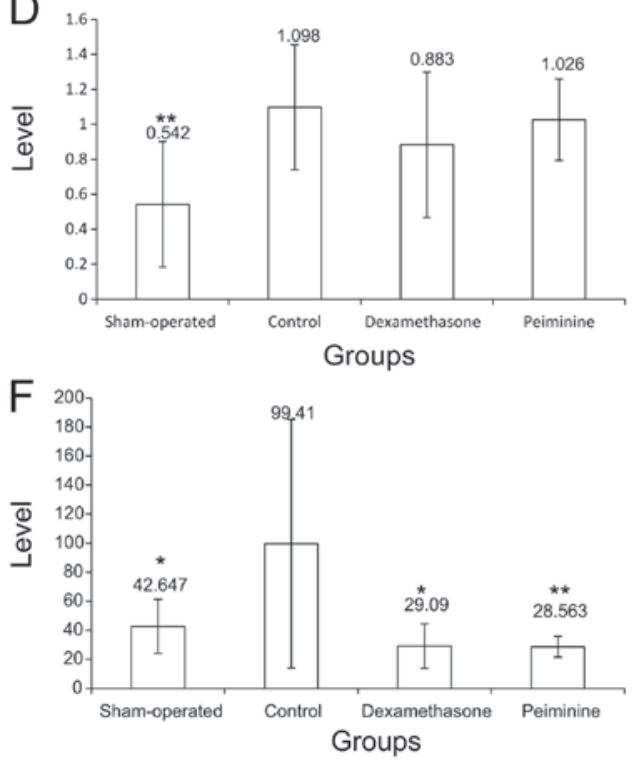

Figure 4. Lung index and serum levels of inflammatory mediators. (A) Body weight measurements in the 4 groups. Body weight was significantly reduced $(\mathrm{P}<0.01)$ in the control, compared to the sham-operated group. The peiminine group had a significantly lower body weight $(\mathrm{P}<0.01)$ compared to the control group. (B) Lung weight measurements in the 4 groups. Lung weight was significantly increased $(\mathrm{P}<0.05)$ in the control, compared to the sham-operated group. The dexamethasone and peiminine groups had significantly lower body weights $(\mathrm{P}<0.01)$ compared to the control group. (C) Lung index measurements in the 4 groups. Lung index was significantly increased $(\mathrm{P}<0.01)$ in the control, compared to the sham-operated group. The peiminine group had a significantly lower lung index $(\mathrm{P}<0.01)$ compared to the control group. (D) Serum IL-4 levels in the 4 groups. IL-4 was significantly elevated in the control (P<0.01), compared to the sham-operated group. IL-4 levels in the dexamethasone and peiminine groups were not significantly different from that of the control group. (E) Serum TNF- $\alpha$ levels in the 4 groups. No statistically significant differences were evident. (F) Serum IFN- $\gamma$ levels in the 4 groups. IFN- $\gamma$ levels were significantly elevated in the control $(\mathrm{P}<0.05)$, compared to the sham-operated group. Levels in the dexamethasone $(\mathrm{P}<0.05)$ and peiminine $(\mathrm{P}<0.01)$ groups were significantly lower compared to the control group. ${ }^{*} \mathrm{P}<0.05 ;{ }^{* *} \mathrm{P}<0.01$.

relatively small amount of collagen fibers was present. In the control group, a substantial increase in the number of collagen fibers was evident, typical of pulmonary fibrosis. Evidence of pulmonary fibrosis was also observed in the DXS and peiminine groups, although to a lesser extent compared to that observed in the control group. 

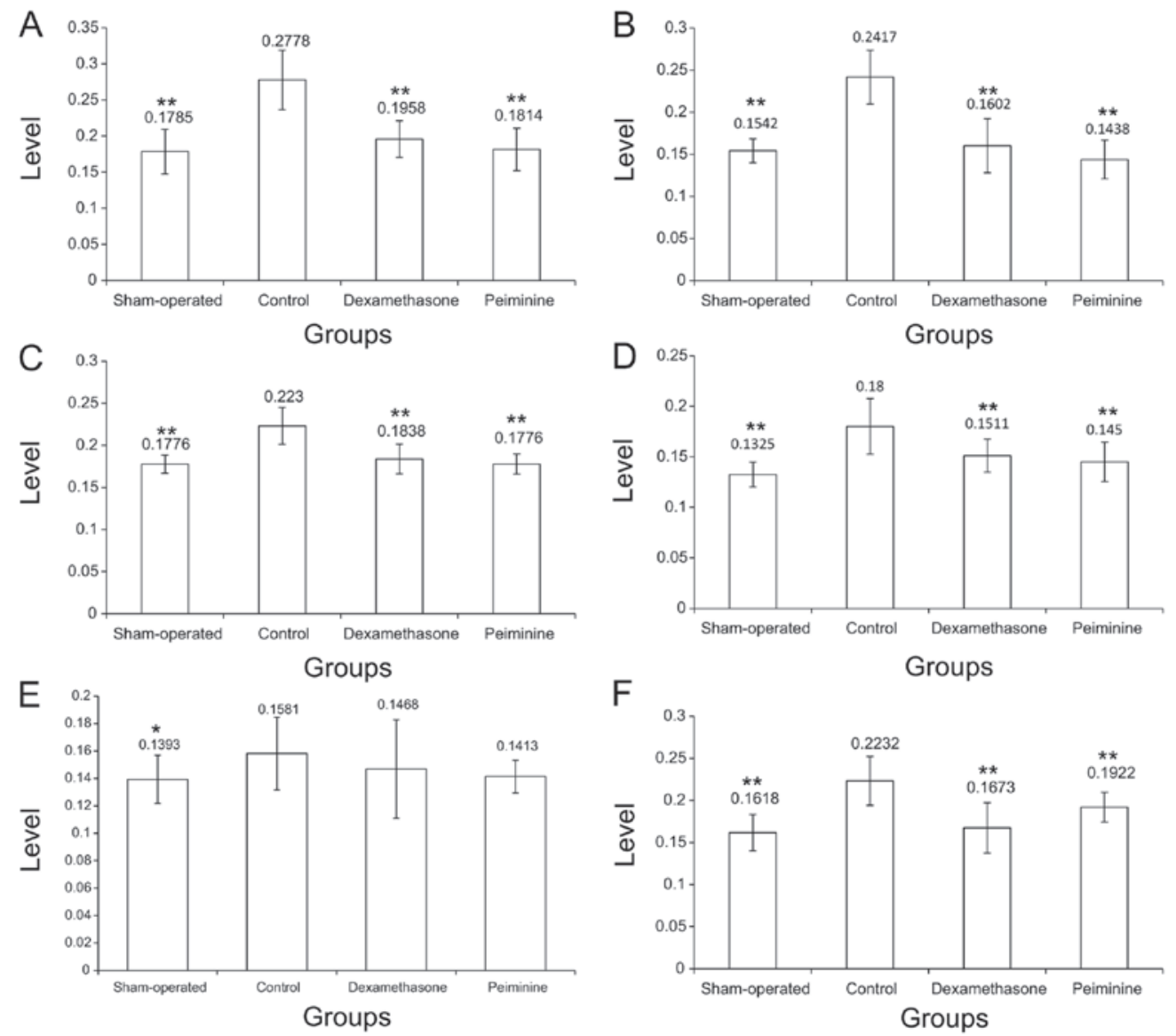

Figure 5. Levels of TGF- $\beta$, CTGF, NF- $\kappa B$, ERK1/2, Fas and FasL in lung tissue. (A) TGF- $\beta$ levels in the 4 groups. TGF- $\beta$ was significantly increased $(\mathrm{P}<0.01)$ in the control, compared to the sham-operated group. The dexamethasone and peiminine groups had significantly lower TGF- $\beta$ levels $(\mathrm{P}<0.01)$ compared to the control group. (B) CTGF levels in the 4 groups. CTGF was significantly increased $(\mathrm{P}<0.01)$ in the control, compared to the sham-operated group. The dexamethasone and peiminine groups had significantly lower CTGF levels $(\mathrm{P}<0.01)$ compared to the control group. $(\mathrm{C}) \mathrm{NF}-\mathrm{kB}$ levels in the 4 groups. NF-kB was significantly increased $(\mathrm{P}<0.01)$ in the control, compared to the sham-operated group. The dexamethasone and peiminine groups had significantly lower NF- $\mathrm{KB}$ levels $(\mathrm{P}<0.01)$ compared to the control group. (D) ERK1/2 levels in the 4 groups. ERK1/2 was significantly elevated in the control $(\mathrm{P}<0.01)$, compared to the sham-operated group. The dexamethasone and peiminine groups had significantly lower ERK1/2 levels $(\mathrm{P}<0.01)$ compared to the control group. (E) Fas levels in the 4 groups. The Fas level in the control group was significantly higher $(\mathrm{P}<0.01)$ compared to that in the sham-operated group, but not significantly different from values in the dexamethasone and peiminine groups. (F) FasL levels in the 4 groups. FasL was significantly elevated in the control $(\mathrm{P}<0.01)$, compared to the sham-operated group. Levels in the dexamethasone and peiminine groups were significantly lower $(\mathrm{P}<0.01)$ compared to the control group. ${ }^{*} \mathrm{P}<0.05 ;{ }^{* * *} \mathrm{P}<0.01$.

Alveolitis and pulmonary fibrosis scores. As shown in Fig. 3, scores for alveolar inflammation and pulmonary fibrosis were significantly higher in rats in the control group compared to rats in the sham-operated group $(\mathrm{P}<0.01)$. Furthermore, the alveolitis and pulmonary fibrosis scores in the DXS and peiminine groups were significantly lower compared to the corresponding scores in the control group $(\mathrm{P}<0.01)$.

Lung index as a measure of lung injury in rats. Fig. 4A-C presents data for body and lung weight as well as lung index for the 4 groups. The control group showed a significantly elevated lung index $(\mathrm{P}<0.01)$ compared to the sham-operated group, which was associated with a significant increase in lung weight $(\mathrm{P}<0.05)$ and a significant decrease in body weight $(\mathrm{P}<0.01)$. This increase in the lung index is indicative of bleomycininduced lung injury in rats of the control group. Furthermore, the peiminine group was found to have a significantly lower lung index $(\mathrm{P}<0.01)$, as well as a significantly lower lung weight $(\mathrm{P}<0.01)$ compared to the control group. These results suggest that peiminine reduced the extent of the lung injury.

Levels of inflammatory cytokines in the blood. As shown in Fig. 4D-F, 28 days after induction of lung injury, levels of IL-4 $(\mathrm{P}<0.01)$ and IFN- $\gamma(\mathrm{P}<0.05)$ were significantly elevated in the control group, compared to the sham-operated group. The levels of TNF- $\alpha$ and IL-4 in the peiminine and DXS groups were not significantly different to the corresponding values in the control group $(\mathrm{P}>0.05)$. However, the levels of IFN- $\gamma$ in the peiminine and DXS groups were significantly lower compared to that of the control group $(\mathrm{P}<0.01)$.

Cell signal transduction pathways. As shown in Fig. 5, the levels of TGF- $\beta$, CTGF, NF- $\kappa$ B, ERK1/2, FasL and Fas were significantly higher in the control group compared to those in the sham-operated group $(\mathrm{P}>0.05$ for Fas; $\mathrm{P}<0.01$ for the others). 
Compared to the control group, the peiminine and DXS groups showed significantly lower levels of TGF- $\beta$, CTGF, NF- $\kappa$ B, ERK1/2 and FasL ( $\mathrm{P}<0.01$ for all). By contrast, no statistically significant differences were observed in these groups for Fas $(\mathrm{P}>0.05)$.

\section{Discussion}

The main findings of our study are that peiminine is as effective as DXS in reducing the degree of alveolitis and the extent of pulmonary fibrosis (assessed using histological scoring methods and the lung index), 28 days after bleomycin-induced lung injury in rats. These effects of peiminine were associated with a reduced level of serum IFN- $\gamma$, and decreased expression of TGF- $\beta$, CTGF, ERK1/2, NF- $\kappa$ B and FasL in lung tissue. The beneficial actions of peiminine may thus be due to the inhibitory effects on these aforementioned mediators, which are known to be involved in the pathogenesis of ALI.

Intratracheal application of bleomycin is a widely used technique for inducing ALI in rodent animal model systems, resulting in an initial development of pulmonary oedema that is followed by a fibrotic interstitial reaction $(20,22,23)$. In the present study, histological comparison of the control (bleomycin) and the sham-operated groups showed clear evidence of inflammation, edema and collagen deposition in lung sections of the control group that were not present in the sham-operated group. Furthermore, the grading of alveolitis and pulmonary fibrosis using established scoring systems demonstrated that bleomycin induced these two pathological changes. In addition, levels of IFN- $\gamma$ and IL-4 in the serum were significantly increased in the control group (compared to the sham-operated group), as were levels of TGF- $\beta$, CTGF, ERK1/2, NF- $\mathrm{KB}$ and FasL in lung tissue. These data clearly indicate that bleomycin successfully induced lung injury, alveolitis and pulmonary fibrosis in the rats used in our study, supporting our use of this method as a model of ALI.

Over the past decade, substantial progress has been made in understanding the pathophysiology of lung fibrosis. The design of successful anti-fibrotic therapies may need to focus on mechanisms or pathways, downstream of the inflammatory process, that mediate fibroproliferation. The identification of intracellular signaling pathways eliciting the cellular responses of mesenchymal cell proliferation and differentiation as well as extracellular matrix deposition, may facilitate the development of novel therapeutic approaches to ameliorate the global burden of fibroproliferative diseases $(9,24)$. Inhibition of signal transduction proteins is now widely acknowledged as a valid strategy to combat inflammatory disease (23). Notably, studies have reported that neferine, methyl palmitate, naringin, astragalin, luteolin and paeonol have inhibitory effects on pulmonary fibrosis, due to their actions as antiinflammatory agents, anti-oxidants and inhibitors of cytokines and NF- $\mathrm{kB}(22,25-30)$. Our study suggests that peiminine may also have such beneficial effects, which are comparable to those of DXS.

The process of fibrosis is promoted by early pro-inflammatory mediators, hence blocking of these mediators may be one approach to attenuate fibrosis. Evidence from several clinical studies has indicated that pro-inflammatory cytokines, notably TNF- $\alpha$, IL-1 and IL-6, participate in the early devel- opment of inflammation and play a crucial role in ALI $(3,4)$. TNF- $\alpha$ is known as a primary cytokine, since it amplifies the inflammatory cascade to cause inflammatory injury and recruits neutrophils into the lung $(5,23)$. Furthermore, IL-4 is an anti-inflammatory cytokine that is able to inhibit the function of TNF- $\alpha$ and reduce inflammatory injury to lung tissue (31). In the present study, serum levels of TNF- $\alpha$ and IL-4 were not significantly affected by peiminine and DXS, whereas the level of IFN- $\gamma$ was reduced. This would suggest that inhibitory actions of peiminine and DXS on alveolitis and pulmonary fibrosis are not secondary to effects on TNF- $\alpha$ and IL-4, but may instead be the consequence, at least in part, of decreased secretion of IFN- $\gamma$.

Previous studies have identified a number of chemokines, cytokines and growth factors that mediate pulmonary fibrosis $(3,4,7)$. Of these, TGF- $\beta 1$ is thought to be one of the key mediators that links inflammation to fibrogenesis. CTGF is a downstream mediator of TGF- $\beta 1$ that induces connective tissue cell proliferation and extracellular matrix deposition (8). It is therefore of note that, in our experiments, peiminine as well as DXS caused reductions in the tissue expression of CTGF and TGF- $\beta$. Upregulation of TGF- $\beta 1$ and CTGF are known to be critically involved in the pathogenesis of pulmonary fibrosis $(23,32)$. TGF- $\beta 1$ is a potent pro-fibrotic factor that plays a pivotal role in several pathological processes, including the transition of alveolar epithelial cells to myofibroblasts (33-35). Consistent with this hypothesis, impaired TGF- $\beta$ responsiveness appears to result in a reduction of fibrosis (32). CTGF has been reported to be useful in diagnosing or predicting disease progression in certain fibrotic diseases (36), while CTGF levels in blood are considered to reflect fibrosis in a variety of organs (37). Furthermore, inhibiting the upregulation of CTGF can attenuate bleomycin-induced ALI and pulmonary fibrosis (23), while certain agents that inhibit bleomycin-induced ALI and pulmonary fibrosis have been reported to act through inhibition of TGF- $\beta 1$ and CTGF. It is therefore reasonable to conclude that some of the inhibitory effects of peiminine (and also DXS) on bleomycin-induced ALI are via reduced expression of CTGF and TGF- $\beta$.

The NF- $\mathrm{B}$ family of transcription factors regulates inflammation, survival, proliferation and other biological processes (12). There are clear links between canonical activation of NF- $\mathrm{KB}$ in immune cells to the pathogenesis of inflammatory diseases (13), and the expression of pro-inflammatory mediators is known to be modulated by NF- $\mathrm{KB}$ (26). Stimulation of the NF- $\kappa \mathrm{B}$ pathway is mediated by diverse signal transduction cascades in response to several stress conditions, such as infection and inflammation. We found that administration of peiminine and DXS were associated with significant reductions in tissue NF- $\mathrm{kB}$ expression. These actions may thus contribute to the protective effects of these agents on ALI.

The MAPK-ERK signaling cascade is a major pathway controlling cellular processes associated with fibrogenesis, including growth, proliferation and survival. In progressive pulmonary fibrosis associated with increased MAPK/ERK activation, ERK has been reported to be primarily activated in the mesenchymal cells of the fibrotic lesions (9). Clinical findings have demonstrated an upregulation of MAPK/ERK 
in human fibrotic disease (10). The MAPK/ERK pathway is a logical target for potential fibrosis therapy, as several fibrogenic cytokines signal through MAPK/ERK (11), and selective inhibition of MAPK prevents the development and attenuates the progression of fibrosis, when administered as a rescue therapy. Our findings that peiminine and DXS cause reduced expression of ERK1/2 in lung tissue indicate that decreased signaling through the MAPK/ERK pathway contributes to the anti-fibrotic effects of these drugs. In addition, actions on FasL, which is also involved in the fibrotic process (38), may also play a role in the effects of peiminine and DXS.

Our study is not without limitations. First, although we have shown that peiminine has protective effects against bleomycin-induced ALI, it cannot be certain that such effects would extend to other chemotherapy agents or to radiationinduced injury. However, the bleomycin model is widely used and validated, hence our data are likely to have applicability to human patients, at least to a certain extent. Second, although changes in the levels of various cytokines and mediators have been identified following peiminine treatment in our rat model system, the primary mediators that contribute to the beneficial actions of peiminine are yet to be identified. In addition, it cannot be definitively concluded that similar changes in mediator levels would be seen in human patients. Additional studies are required to expand upon our observations, and describe the mechanisms underlying the actions of peiminine more precisely.

In conclusion, our findings indicate that peiminine has beneficial effects protecting against bleomycin-induced lung injury in rats, and that these effects are comparable to those of DXS. Furthermore, the attenuation of pulmonary fibrosis by peiminine is associated with a reduction in the levels of IFN- $\gamma$ in the blood, and CTGF, TGF- $\beta, \mathrm{NF}-\kappa \mathrm{B}, \mathrm{ERK} 1 / 2$ and FasL in lung tissue. Thus, our findings provide evidence that peiminine may have therapeutic potential in the treatment of ALI and pulmonary fibrosis.

\section{Acknowledgements}

This study was financially supported by the Nature Science Foundation of the Jiangsu province of China (no. 08KJB360008) and the Chinese Postdoctoral Station of the Nanjing Medical University (no. 201102170C).

\section{References}

1. Limper AH: Chemotherapy-induced lung disease. Clin Chest Med 25: 53-64, 2004

2. Graves PR, Siddiqui F, Anscher MS and Movsas B: Radiation pulmonary toxicity: from mechanisms to management. Semin Radiat Oncol 20: 201-207, 2010.

3. Bhatia M, Zemans RL and Jeyaseelan S: Role of chemokines in the pathogenesis of acute lung injury. Am J Respir Cell Mol Biol 46: 566-572, 2012

4. Martin TR and Matute-Bello G: Experimental models and emerging hypotheses for acute lung injury. Crit Care Clin 27: 735-752, 2011

5. Mukhopadhyay S, Hoidal JR and Mukherjee TK: Role of TNFalpha in pulmonary pathophysiology. Respir Res 7: 125, 2006

6. Anscher MS: Targeting the TGF-beta1 pathway to prevent norma tissue injury after cancer therapy. Oncologist 15: 350-359, 2010.

7. Wilson MS, Madala SK, Ramalingam TR, et al: Bleomycin and IL-1beta-mediated pulmonary fibrosis is IL-17A dependent. J Exp Med 207: 535-552, 2010
8. Blom IE, Goldschmeding R and Leask A: Gene regulation of connective tissue growth factor: new targets for antifibrotic therapy? Matrix Biol 21: 473-482, 2002.

9. Madala SK, Schmidt S, Davidson C, Ikegami M, Wert S and Hardie WD: MEK-ERK pathway modulation ameliorates pulmonary fibrosis associated with epidermal growth factor receptor activation. Am J Respir Cell Mol Biol 46: 380-388, 2012.

10. Antoniou KM, Margaritopoulos GA, Soufla G, et al: Expression analysis of Akt and MAPK signaling pathways in lung tissue of patients with idiopathic pulmonary fibrosis (IPF). J Recept Signal Transduct Res 30: 262-269, 2010

11. Jia X, Liu B, Shi X, Ye M, Zhang F and Liu H: Roles of the ERK, JNK/AP-1/cyclin D1-CDK4 pathway in silica-induced cell cycle changes in human embryo lung fibroblast cells. Cell Biol Int 35: 697-704, 2011.

12. Morris GF: An alternative to lung inflammation and fibrosis. Am J Pathol 176: 2595-2598, 2010.

13. Vallabhapurapu S and Karin M: Regulation and function of NF-kappaB transcription factors in the immune system. Annu Rev Immunol 27: 693-733, 2009.

14. Johnson ER and Matthay MA: Acute lung injury: epidemiology, pathogenesis, and treatment. J Aerosol Med Pulm Drug Deliv 23: 243-252, 2010

15. Lamontagne F, Briel M, Guyatt GH, Cook DJ, Bhatnagar N and Meade M: Corticosteroid therapy for acute lung injury, acute respiratory distress syndrome, and severe pneumonia: a meta-analysis of randomized controlled trials. J Crit Care 25: 420-435, 2010.

16. Raghu G, Collard HR, Egan JJ, et al: An official ATS/ERS/JRS/ ALAT statement: idiopathic pulmonary fibrosis: evidence-based guidelines for diagnosis and management. Am J Respir Crit Care Med 183: 788-824, 2011.

17. Wang D, Zhu J, Wang S, et al: Antitussive, expectorant and anti-inflammatory alkaloids from Bulbus Fritillariae Cirrhosae. Fitoterapia 82: 1290-1294, 2011.

18. Wang D, Wang S, Chen X, et al: Antitussive, expectorant and antiinflammatory activities of four alkaloids isolated from Bulbus of Fritillaria wabuensis. J Ethnopharmacol 139: 189-193, 2012.

19. Taooka Y, Maeda A, Hiyama K, Ishioka S and Yamakido M: Effects of neutrophil elastase inhibitor on bleomycin-induced pulmonary fibrosis in mice. Am J Respir Crit Care Med 156: 260-265, 1997.

20. Aono Y, Nishioka Y, Inayama M, et al: Imatinib as a novel antifibrotic agent in bleomycin-induced pulmonary fibrosis in mice. Am J Respir Crit Care Med 171: 1279-1285, 2005.

21. Szapiel SV, Elson NA, Fulmer JD, Hunninghake GW and Crystal RG: Bleomycin-induced interstitial pulmonary disease in the nude, athymic mouse. Am Rev Respir Dis 120: 893-899, 1979.

22. El-Demerdash E: Anti-inflammatory and antifibrotic effects of methyl palmitate. Toxicol Appl Pharmacol 254: 238-244, 2011.

23. Kim JW, Rhee CK, Kim TJ, et al: Effect of pravastatin on bleomycin-induced acute lung injury and pulmonary fibrosis. Clin Exp Pharmacol Physiol 37: 1055-1063, 2010.

24. Hardie WD, Glasser SW and Hagood JS: Emerging concepts in the pathogenesis of lung fibrosis. Am J Pathol 175: 3-16, 2009.

25. Zhao L, Wang X, Chang Q, et al: Neferine, a bisbenzylisoquinline alkaloid attenuates bleomycin-induced pulmonary fibrosis. Eur J Pharmacol 627: 304-312, 2010.

26. Liu Y, Wu H, Nie YC, Chen JL, Su WW and Li PB: Naringin attenuates acute lung injury in LPS-treated mice by inhibiting NF-кB pathway. Int Immunopharmacol 11: 1606-1612, 2011.

27. Lee HB, Kim EK, Park SJ, Bang SG, Kim TG and Chung DW: Isolation and anti-inflammatory effect of astragalin synthesized by enzymatic hydrolysis of tea seed extract. J Sci Food Agric 91: 2315-2321, 2011 .

28. Fu PK, Wu CL, Tsai TH and Hsieh CL: Anti-inflammatory and anticoagulative effects of paeonol on LPS-induced acute lung injury in rats. Evid Based Complement Alternat Med 2012: 837513, 2012

29. Lee JP, Li YC, Chen HY, et al: Protective effects of luteolin against lipopolysaccharide-induced acute lung injury involves inhibition of MEK/ERK and PI3K/Akt pathways in neutrophils. Acta Pharmacol Sin 31: 831-838, 2010.

30. Soromou LW, Chen N, Jiang L, et al: Astragalin attenuates lipopolysaccharide-induced inflammatory responses by downregulating NF- $\kappa \mathrm{B}$ signaling pathway. Biochem Biophys Res Commun 419: 256-261, 2012

31. Levings MK and Schrader JW: IL-4 inhibits the production of TNF-alpha and IL-12 by STAT6-dependent and -independent mechanisms. J Immunol 162: 5224-5229, 1999. 
32. Carey WA, Taylor GD, Dean WB and Bristow JD: Tenascin-C deficiency attenuates TGF- $\beta$-mediated fibrosis following murine lung injury. Am J Physiol Lung Cell Mol Physiol 299: L785-L793, 2010.

33. Acloque H, Adams MS, Fishwick K, Bronner-Fraser M and Nieto MA: Epithelial-mesenchymal transitions: the importance of changing cell state in development and disease. J Clin Invest 119: 1438-1449, 2009.

34. Xu J, Lamouille S and Derynck R: TGF-beta-induced epithelial to mesenchymal transition. Cell Res 19: 156-172, 2009.

35. Ramos C, Becerril C, Montano M, et al: FGF-1 reverts epithelial-mesenchymal transition induced by TGF- $\{$ beta $\} 1$ through MAPK/ERK kinase pathway. Am J Physiol Lung Cell Mol Physiol 299: L222-L231, 2010.
36. Kono M, Nakamura Y, Suda T, et al: Plasma CCN2 (connective tissue growth factor; CTGF) is a potential biomarker in idiopathic pulmonary fibrosis (IPF). Clin Chim Acta 412: 2211-2215, 2011.

37. Leask A, Parapuram SK, Shi-Wen $X$ and Abraham DJ: Connective tissue growth factor (CTGF, CCN2) gene regulation: a potent clinical bio-marker of fibroproliferative disease? J Cell Commun Signal 3: 89-94, 2009.

38. Dosreis GA, Borges VM and Zin WA: The central role of Fas-ligand cell signaling in inflammatory lung diseases. J Cell Mol Med 8: 285-293, 2004. 